OPEN ACCESS

Edited by:

Johanna Ospel,

University Hospital of

Basel, Switzerland

Reviewed by:

Fouzi Bala,

University of Calgary, Canada

Silke Walter,

Saarland University Hospital, Germany

*Correspondence:

Elin Wiebert

elin.wiebert@stud.ki.se

Specialty section:

This article was submitted to

Stroke,

a section of the journa

Frontiers in Neurology

Received: 26 August 2021

Accepted: 28 October 2021

Published: 29 November 2021

Citation:

Wiebert E, Berglund A, Sjöstrand C,

Eriksson EE and Mazya MV (2021)

Sex Equitable Prehospital Stroke Triage Using Symptom Severity and

Teleconsultation.

Front. Neurol. 12:765296.

doi: 10.3389/fneur.2021.765296

\section{Sex Equitable Prehospital Stroke Triage Using Symptom Severity and Teleconsultation}

\author{
Elin Wiebert ${ }^{1 *}$, Annika Berglund ${ }^{1,2}$, Christina Sjöstrand ${ }^{1,3}$, Einar E. Eriksson ${ }^{1,2}$ and \\ Michael V. Mazya ${ }^{1,2}$ \\ ${ }^{1}$ Department of Clinical Neuroscience, Karolinska Institutet, Stockholm, Sweden, ${ }^{2}$ Department of Neurology, Karolinska \\ University Hospital, Stockholm, Sweden, ${ }^{3}$ Department of Neurology, Danderyd Hospital, Stockholm, Sweden
}

Objectives: We aimed to determine whether there are sex differences in prehospital accuracy of the Stockholm Stroke Triage System (SSTS) to predict large artery occlusion $(\llcorner A O)$ stroke, and endovascular thrombectomy (EVT), and whether clinical characteristics differ between men and women undergoing "code stroke" ambulance transport.

Materials and Methods: This prospective observational study collected data between October 2017 and October 2018. We included 2,905 patients, transported as "code stroke," by nurse-staffed ground ambulance, to a Stockholm Region hospital. Exclusion criteria were private or helicopter transport, onset outside Stockholm, and in-hospital stroke. We compared overall accuracy, sensitivity, specificity, positive and negative predictive values, and clinical characteristics between sexes.

Results: No significant sex differences in SSTS predictive performance for LAO or EVT were found, overall accuracy for LAO $87.3 \%$ in women vs. $86.7 \%$ in men. Women were median 4 years older and more frequently had stroke mimics (46.2 vs. $41.8 \%$ ). Women more commonly had decreased level of consciousness (14.0 vs. $10.2 \%)$ and moderate-to-severe motor symptoms (by 2.7-3.8 percentage points), and less commonly limb ataxia (7.2 vs. 9.7\%).

Conclusions: The SSTS had equal predictive performance for LAO and EVT among men and women, despite minor sex differences in the clinical characteristics in patients undergoing ambulance transport for suspected stroke.

Keywords: acute ischemic stroke, thrombectomy, triage, telemedicine, sex characteristics

\section{INTRODUCTION}

In 2015, clinical trials established the superiority of endovascular thrombectomy (EVT) over medical treatment in large artery occlusion (LAO) stroke (1). However, EVT is only available at certain hospitals and its benefits diminish rapidly with time, accentuating the need for an accurate prehospital triage.

The Stockholm Region in Sweden has a 2.3 million population, across $6,519 \mathrm{~km}^{2}$. The region is served by one comprehensive stroke center (CSC), Karolinska University Hospital, and six primary stroke centers (PSC). Intravenous thrombolysis (IVT) and stroke unit care are provided at all stroke centers, while EVT is only available at the CSC. 
Before October 10, 2017, guidelines in Stockholm-mandated code stroke, priority 1 ambulance transport for patients with positive modified face-arm-speech-time (FAST) test or other cause for stroke suspicion raised by the ambulance nurse, presented within $6 \mathrm{~h}$ of onset, to the most proximal stroke unit. Patients presenting beyond $6 \mathrm{~h}$ of onset, or with unknown time of onset, were also transported with code stroke if displaying critically affected vital signs. On hospital arrival, patients were examined using plain $\mathrm{CT}$ and, in eligible patients, IVT was administered. Thereafter, CT angiography (CTA) was performed, unless contraindicated by local hospital guidelines or on clinical grounds. Reasons to abstain from CTA included minor symptoms with $>8 \mathrm{~h}$ since the last known well, demarcated CT infarct findings fully matching the clinical presentation, contraindications to EVT, such as modified Rankin Scale (mRS) four to five or prestroke life expectancy below 3 months, and contraindications to CTA, such as severe renal failure or contrast allergy. This routine remained unchanged after the Stockholm Stroke Triage System (SSTS) implementation in 2017. When anterior or posterior circulation LAO was found on CTA at the PSC, a CSC stroke physician, with access to electronically transferred images, was teleconsulted on the patient eligibility for transfer for EVT. Reasons for declining transfer were infarct changes $>$ onethird of the middle cerebral artery or greater than one-half of another territory on plain CT, prestroke mRS score 4-5, prestroke life expectancy $<3$ months, or CTA results or medical history indicating severe catheter access difficulties. However, age and time since onset or last known well, did not alone affect transfer decisions. Regional quality registry data showed that onset-to-puncture time was $1-2 \mathrm{~h}$ longer in secondary transfers, which constituted $75 \%$ of the patients treated with EVT, compared with patients directly transported to the CSC when it was most geographically proximal to their place of symptom onset.

The SSTS combines a test for moderate-to-severe hemiparesis with ambulance-hospital teleconsultation, to identify patients with a high likelihood of LAO with EVT indication, for PSC bypass. The SSTS predicts LAO and EVT with high accuracy both within 6 and $6-24 \mathrm{~h}$ from onset or last known well, and has reduced regional median onset-to-puncture time in EVT by $69 \mathrm{~min}$, without delaying IVT $(2,3)$. Studies on AIS have found that, unlike greater mortality, the lower poststroke functional level and quality of life in women remains significant after adjustment for prestroke mRS and age (4-6). To better understand these disparities, research has focused on sex differences in clinical characteristics in stroke. Among patients treated with IVT, women more often have anterior circulation LAO and strokes of greater severity, more frequently caused by cardioembolism because of the atrial fibrillation (7). Traditional stroke symptoms, including hemiparesis, are more common in men compared with women, and the opposite for non-traditional symptoms, such as mental status changes, loss of consciousness, and generalized weakness $(7,8)$. Presenting with non-traditional symptoms might be associated with a greater risk of misdiagnosis (9). Similarly, the greater frequency of stroke mimics in women could add to differences in diagnostic accuracy (7). It is unknown how any sex differences in the clinical characteristics might affect prehospital LAO triage accuracy.

We aimed to determine whether SSTS triage accuracy for LAO and EVT differs between sexes, and if there are sex differences in the clinical characteristics among patients undergoing code stroke ambulance transport.

\section{MATERIALS AND METHODS}

This observational study used data prospectively collected between October 2017 and October 2018, in patients undergoing code stroke ground ambulance transportation to a Stockholm Region hospital. Exclusion criteria were private or helicopter transportation, onset outside the Stockholm Region, and inhospital stroke. Of 2,909 eligible patients, four opted out from the study participation, leaving 2,905 patients in the dataset.

Research Ethics Committee approval was obtained (approval 2017/374) with waived need for active consent. Patients were informed in writing of their right to decline study data collection.

On October 10, 2017, the novel SSTS, using a three-step algorithm, was implemented across the region. In step 1, stroke suspicion was raised by an ambulance nurse, due to positive modified FAST test or other clinical reasons. In step 2, stroke suspects were prehospitally assessed using an NIH Stroke Scale (NIHSS)-derived test for moderate-to-severe hemiparesis, defined as a score of $\geq 2$ NIHSS points each in the ipsilateral arm and leg (the A2L2 test). Test status was classified as positive or negative. The test was inapplicable in the patients presenting with seizures, unconsciousness or bilateral paresis. In step 3, a CSC stroke physician was consulted by telephone on A2L2 positive cases, to discuss the diagnostic suspicion and assess EVT eligibility. This was followed by the destination decision, frequently directly to the CSC, bypassing any more proximal PSC(s). The region-wide electronic health record system allowed CSC physicians to assess prestroke mRS scores of the patients and comorbidities. In A2L2 negative or A2L2 inapplicable cases, a stroke physician at the most proximal hospital (most frequently a PSC) was prenotified of subsequent transportation of the patients there.

The SSTS guideline and protocol, including a patient flow chart (Figure 1), was published on the Stockholm Healthcare Region website, and programmed into ambulance portable tablet computers. All the regional ambulances have a crew of two: at least one specialist ambulance nurse (3-year university degree and 1 year of prehospital or anesthesia training) and an ambulance technician (nursing high school diploma and professional training). Prior to implementation, ambulance nurses had web-based training and live lectures, and hospital staff underwent group training.

Triage positive status was defined as suspected stroke with positive A2L2 test and acceptance for direct transport to the CSC following teleconsultation between an ambulance nurse and a hospital physician. Patients testing A2L2 negative or inapplicable, and those testing A2L2 positive but declined PSC bypass because of the EVT contraindications, were classified 


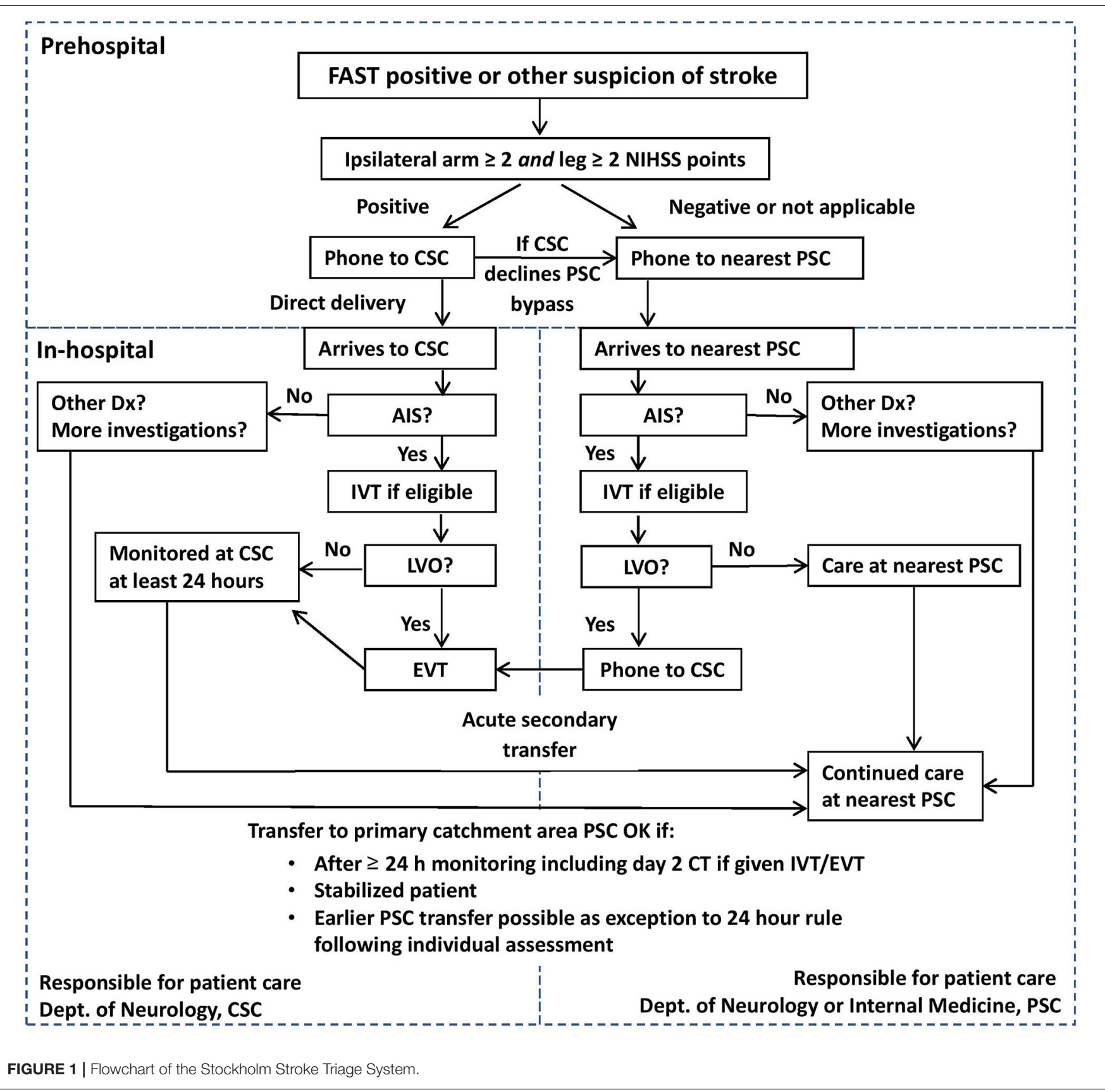

as triage negative. Reasons for A2L2 positive patients to be declined PSC bypass were low suspicion of stroke, prestroke mRS 4-5, prestroke life expectancy $<3$ months and critically affected vital signs requiring stabilization at the nearest hospital. The same classification principles were applied when the CSC was the most proximal hospital, defining triage positivity as confirmed stroke suspicion, A2L2 positivity and absence of contraindications to EVT. LAO stroke was defined as acute ischemic stroke (AIS) with CTA-confirmed occlusion or subocclusion in the arterial segments routinely treated at the CSC: ICA, M1-2, A1-2, P1, BA, and intracranial VA.
In the patients with contraindications to CTA, LAO stroke was defined as a dense cerebral artery on plain CT. For analyses of triage accuracy, patients with AIS not undergoing CTA were pooled with patients with CTA confirmed nonLAO AIS. Diagnostic imaging was routinely evaluated by two radiologists. EVT was defined as arterial puncture. Final diagnoses, including mimic diagnoses, as well as stroke and TIA diagnoses, were established during in-hospital care and were obtained from the final discharge notes signed by the senior consultant-level physician responsible for the in-hospital care period. 
TABLE 1 | Clinical characteristics in men and women.

\begin{tabular}{|c|c|c|c|c|c|}
\hline \multirow[b]{2}{*}{ Characteristic } & \multicolumn{2}{|c|}{$\begin{array}{c}\text { Men } \\
(n=1,485)\end{array}$} & \multicolumn{2}{|c|}{$\begin{array}{c}\text { Women } \\
(n=1,420)\end{array}$} & \multirow[t]{2}{*}{$P$} \\
\hline & No./total & $\begin{array}{c}\text { Median (IQR) } \\
\text { or } \%\end{array}$ & No./total & $\begin{array}{c}\text { Median (IQR) } \\
\text { or } \%\end{array}$ & \\
\hline Age & $1,485 / 1,485$ & $74(64-81)$ & $1,420 / 1,420$ & 78 (66-86) & $<0.001$ \\
\hline A2L2 positive & $260 / 1,485$ & 17.5 & $270 / 1,420$ & 19.0 & 0.294 \\
\hline Triage positive & $168 / 1,485$ & 11.3 & $155 / 1,420$ & 10.9 & 0.733 \\
\hline CTA performed & $712 / 1,484$ & 48.0 & $626 / 1,419$ & 44.1 & 0.037 \\
\hline $\mathrm{NIHSS}$ total & $1,298 / 1,485$ & $4(1-9)$ & $1,221 / 1,420$ & $4(1-10.5)$ & 0.007 \\
\hline \multicolumn{6}{|l|}{ NIHSS items $\left(>0 \mathrm{p}^{\dagger}\right)$} \\
\hline 1a. LOC & $123 / 1,207$ & 10.2 & $158 / 1,127$ & 14.0 & 0.005 \\
\hline 1b. LOC questions & $422 / 1,201$ & 35.1 & $433 / 1,122$ & 38.6 & 0.085 \\
\hline 1c. LOC commands & $180 / 1,194$ & 15.1 & $220 / 1,116$ & 19.7 & 0.003 \\
\hline 2. Gaze & $157 / 1,194$ & 13.1 & $168 / 1,110$ & 15.1 & 0.171 \\
\hline 3. Visual & $171 / 1,187$ & 14.4 & 173/1,099 & 15.7 & 0.372 \\
\hline 4. Facial palsy & $420 / 1,196$ & 35.1 & $410 / 1,116$ & 36.7 & 0.417 \\
\hline 5a. Arm, left, $\geq 2 p$ & $165 / 1,202$ & 13.7 & $196 / 1,120$ & 17.5 & 0.012 \\
\hline 5b. Arm, right, $\geq 2 p$ & $144 / 1,201$ & 12.0 & $164 / 1,119$ & 14.7 & 0.059 \\
\hline 6a. Leg, left, $\geq 2 \mathrm{p}$ & $177 / 1,200$ & 14.8 & $207 / 1,124$ & 18.4 & 0.017 \\
\hline 6b. Leg, right, $\geq 2 p$ & $160 / 1,200$ & 13.3 & $188 / 1,118$ & 16.8 & 0.010 \\
\hline 7. Limb ataxia & $114 / 1,179$ & 9.7 & $79 / 1,093$ & 7.2 & 0.037 \\
\hline 8. Sensory & $289 / 1,191$ & 24.3 & 283/1,098 & 25.8 & 0.405 \\
\hline 9. Best language & $388 / 1,199$ & 32.4 & $375 / 1,113$ & 33.7 & 0.496 \\
\hline 10. Dysarthria & $447 / 1,195$ & 37.4 & $399 / 1,110$ & 35.9 & 0.467 \\
\hline 11. Extinction or inattention & $119 / 1,174$ & 10.1 & $137 / 1,091$ & 12.6 & 0.069 \\
\hline \multicolumn{6}{|l|}{ Diagnosis } \\
\hline AIS with LAO & $163 / 1,485$ & 11.0 & $153 / 1,420$ & 10.8 & 0.861 \\
\hline AIS without LAO & $295 / 1,485$ & 19.9 & $229 / 1,420$ & 16.1 & 0.008 \\
\hline AIS, LAO unknown & $226 / 1,485$ & 15.2 & $231 / 1,420$ & 16.3 & 0.438 \\
\hline Intracranial hemorrhage & $181 / 1,485$ & 12.2 & $151 / 1,420$ & 10.6 & 0.188 \\
\hline Stroke mimic & $620 / 1,485$ & 41.8 & $656 / 1,420$ & 46.2 & 0.016 \\
\hline \multicolumn{6}{|l|}{ Treatment } \\
\hline EVT & $64 / 1,485$ & 4.3 & $55 / 1,420$ & 3.9 & 0.553 \\
\hline IVT & $179 / 1,485$ & 12.1 & $158 / 1,420$ & 11.1 & 0.435 \\
\hline OPT, min (EVT) & $64 / 1,485$ & $285(121-373)$ & $55 / 1,420$ & 299 (134-325) & 0.378 \\
\hline ONT, min (IVT) & $176 / 1,485$ & $122(81-167)$ & $156 / 1,420$ & $124(81-160)$ & 0.847 \\
\hline
\end{tabular}

A2L2 indicates $\geq 2$ National Institutes of Health Stroke Scale points each in both the ipsilateral extremities; AIS, acute ischemic stroke; CTA, CT angiography; IQR, interquartile range; IVT, IV thrombolysis; LAO, large artery occlusion; LOC, level of consciousness; NIHSS, NIH Stroke Scale; No., number; ONT, Onset-to-Needle (thrombolysis) Time; OPT, Onset-to-arterial puncture time; $p$, points.

${ }^{+}$All NIHSS items categorized as positive if scoring $>0$ points, except motor items 5 and 6 , which were categorized as $\geq 2$ vs. 0-1 points.

Triage accuracy measures were sensitivity, specificity, positive and negative predictive values (PPV, NPV), and overall accuracy. Clinical characteristics included sex, age, A2L2 and triage status, use of CTA, site of occlusion or thrombosis, NIHSS total score and subitem scores, final diagnosis, onsetto-needle time (ONT) in IVT and onset-to-puncture time (OPT) in EVT. Pearson's chi-squared test and Mann-Whitney $U$-test were used for comparative analyses of categorical and continuous variables. Two-sided $P$ values of $<0.05$ were considered statistically significant. All the analyses were conducted using IBM SPSS version 27 (IBM Corp., Armonk, NY, USA).

\section{RESULTS}

Among 2,905 code stroke ambulance-transported patients, 1,420 (48.9\%) were women. Clinical characteristics are presented in Table 1. Women were older than men, median 78 vs. 74 years. There were no significant sex differences in triage positive status (10.9 vs. $11.3 \%)$ or A2L2 positive test (19.0 vs. $17.5 \%)$. LAO stroke diagnosis was made in 10.8 and $11.0 \%$ in women and men, respectively. Women more frequently received a stroke mimic diagnosis, 46.2 vs. $41.8 \%$. CTA was less frequently used in women, 44.1 vs. $48.0 \%$. While the overall distribution of occlusion sites showed a statistically significant difference between the sexes 
(Table 2), the absolute differences were modest, e.g., MCA M1 occlusion in $11.7 \%$ of women vs. $8.1 \%$ of men. Table 3 shows nearly identical performance of the SSTS in both the sexes, regarding the prediction of LAO and EVT. Overall accuracy for LAO was 87.3 vs. $86.7 \%$, and for EVT 90.7 vs. $90.4 \%$ in women and men respectively, with similar nonsignificant magnitudes of difference in sensitivity, specificity, NPVs, and PPVs.

Out of 2,905 cases, 2,519 (86.7\%) cases with available hospital NIHSS scores, the median total was 4 points in both the sexes (Table 1). Regarding NIHSS subitems (Table 1), women

TABLE 2 | Site of occlusion or thrombosis.

\begin{tabular}{|c|c|c|c|c|c|}
\hline & \multicolumn{2}{|c|}{$\begin{array}{c}\text { Men } \\
(n=1,485)\end{array}$} & \multicolumn{2}{|c|}{$\begin{array}{c}\text { Women } \\
(n=1,420)\end{array}$} & \multirow[t]{2}{*}{$P$} \\
\hline & No./total & $\%$ & No./total & $\%$ & \\
\hline Occlusion site f $^{\ddagger}$ & & & & & 0.001 \\
\hline ICA, extradural only & $12 / 718$ & 1.7 & $5 / 640$ & 0.8 & 0.141 \\
\hline ICA-T & $11 / 718$ & 1.5 & $17 / 640$ & 2.7 & 0.146 \\
\hline $\mathrm{ICA}+\mathrm{MCA}$ & $11 / 718$ & 1.5 & $5 / 640$ & 0.8 & 0.201 \\
\hline M1 & $58 / 718$ & 8.1 & $75 / 640$ & 11.7 & 0.024 \\
\hline M2 & $47 / 718$ & 6.5 & $43 / 640$ & 6.7 & 0.898 \\
\hline M3 or more distal & $5 / 718$ & 0.7 & $5 / 640$ & 0.8 & 0.855 \\
\hline ACA & $2 / 718$ & 0.3 & $4 / 640$ & 0.6 & 0.337 \\
\hline PCA & $8 / 718$ & 1.1 & $13 / 640$ & 2.0 & 0.172 \\
\hline Basilar & $10 / 718$ & 1.4 & $3 / 640$ & 0.5 & 0.081 \\
\hline Vertebral & $16 / 718$ & 2.2 & $4 / 640$ & 0.6 & 0.014 \\
\hline No occlusion or thrombosis & $538 / 718$ & 74.9 & $466 / 640$ & 72.8 & 0.375 \\
\hline
\end{tabular}

${ }^{\ddagger}$ On CTA, or, in patients with contraindications to CTA, corresponding to the dense vessel on CT.

TABLE 3 | Triage accuracy for prediction of LAO and EVT.

\begin{tabular}{|c|c|c|c|c|c|}
\hline \multirow[t]{2}{*}{ Outcome } & \multicolumn{2}{|c|}{$\begin{array}{c}\text { Men } \\
(n=1,485)\end{array}$} & \multicolumn{2}{|c|}{$\begin{array}{c}\text { Women } \\
(n=1,420)\end{array}$} & \multirow[t]{2}{*}{$P$} \\
\hline & No./total. & $\%(95 \% \mathrm{Cl})$ & No./total & $\%(95 \% \mathrm{Cl})$ & \\
\hline \multicolumn{6}{|c|}{ LAO diagnosis } \\
\hline Sensitivity & $67 / 163$ & $41.1(33.6-48.7)$ & $64 / 153$ & $41.8(34.0-49.6)$ & 0.896 \\
\hline Specificity & 1,221/1,322 & 92.4 (90.9-93.8) & $1,176 / 1,267$ & $92.8(91.4-94.2)$ & 0.657 \\
\hline PPV & $67 / 168$ & $39.9(32.5-47.3)$ & $64 / 155$ & $41.3(33.5-49.0)$ & 0.797 \\
\hline NPV & $1,221 / 1,317$ & $92.7(91.3-94.1)$ & $1,176 / 1,265$ & $93.0(91.6-94.4)$ & 0.803 \\
\hline $\begin{array}{l}\text { Overall } \\
\text { accuracy }\end{array}$ & $1,288 / 1,485$ & $86.7(85.0-88.5)$ & $1,240 / 1,420$ & $87.3(85.6-89.1)$ & 0.636 \\
\hline \multicolumn{6}{|c|}{ EVT treatment } \\
\hline Sensitivity & $45 / 63$ & $70.3(59.1-81.5)$ & $39 / 55$ & 70.9 (58.9-82.9) & 0.943 \\
\hline Specificity & $1,298 / 1,421$ & $91.3(89.9-92.8)$ & $1,249 / 1,365$ & $91.5(90.0-93.0)$ & 0.882 \\
\hline PPV & 45/168 & $26.8(20.1-33.5)$ & 39/155 & $25.2(18.3-32.0)$ & 0.740 \\
\hline NPV & $1,298 / 1,317$ & 98.6 (97.9-99.2) & $1,249 / 1,265$ & $98.7(98.1-99.4)$ & 0.696 \\
\hline $\begin{array}{l}\text { Overall } \\
\text { accuracy }\end{array}$ & $1,343 / 1,485$ & 90.4 (88.9-91.9) & $1,288 / 1,420$ & $90.7(89.2-92.2)$ & 0.806 \\
\hline
\end{tabular}

$\mathrm{Cl}$ indicates confidence interval; EVT, endovascular thrombectomy; LAO, large artery occlusion; NPV, negative predictive value; PPV, positive predictive value. somewhat more commonly had decreased level of consciousness (14.0 vs. $10.2 \%)$, affected ability to follow commands (19.7 vs. $15.1 \%$ ), and more moderate-to-severe extremity motor deficits, reaching statistical significance in three out of four extremities (left arm 17.5 vs. $13.7 \%$; left leg 18.4 vs. $14.8 \%$; and right leg 16.8 vs. 13.3\%). Conversely, women had a slightly lower frequency of limb ataxia, 7.2 vs. $9.7 \%$.

\section{DISCUSSION}

We found no sex differences in the prehospital accuracy of the SSTS to predict LAO stroke or EVT. While numerous prehospital LAO stroke triage algorithms have been published (10), we have found no publications on sex differences in this setting. Our results are consistent with a study of stroke triage in an urban, academic Emergency Department, and a study evaluating sex differences in a hub-and-spoke hospital telemedicine system $(11,12)$. Meanwhile, a study in the two US counties in 200507 showed somewhat lower sensitivity for prehospital stroke recognition in women (13).

The near-identical performance of our severity- and teleconsultation-based triage system in both the sexes is consistent with the similarity between men and women regarding hemiparalysis, a symptom strongly associated with the presence of LAO stroke $(2,14)$. Although statistically significant, sex differences in the clinical characteristics among prehospital code stroke patients were minor. We found a somewhat higher frequency of decreased level of consciousness in women upon hospital arrival. This could potentially have affected prehospital triage precision in women negatively, as loss of consciousness has been described as a non-traditional stroke symptom associated with risk of misdiagnosis $(7,9)$. Meanwhile, a minor such effect could have been offset by slightly more common moderateto-severe extremity weakness in women, a traditional stroke symptom potentially facilitating correct stroke identification.

Our finding of more stroke mimics in women with suspected stroke is consistent with previous studies $(7,13,15)$. This, however, did not apparently detract from the triage performance of the system for LAO stroke or EVT. Our finding of lower use of CTA in women deserves mention. Previous sex difference studies of imaging investigations in stroke have also shown a certain disadvantage for women in the crude analysis, which, however, was no longer evident after adjustment for the higher average age in women at stroke onset (7). The association between age and prestroke functional level could, in part, explain this, as premorbid mRS score $4-5$ might in some cases have been considered a contraindication to CTA (since it may have been perceived by the managing physician as not leading to any change of care irrespective of findings) (16). Considering the association between age and comorbidity, and the greater frequency of hypertension among women with AIS, a lower CTA eligibility in women might be caused by a somewhat greater frequency of severe renal failure (17). Unfortunately, our data lack the desired granularity to pursue this question further. Furthermore, with a higher proportion of mimics in women, some of these mimicking conditions will have 
been diagnosed immediately upon arrival at the Emergency Department, and in such cases, no CTA would have been ordered. Furthermore, some mimics would have been diagnosed on an initial plain CT (e.g., intracerebral tumors), with the radiologist actively choosing to abstain from an arterial-phase CTA, and instead do a non-arterial phase contrast-enhanced CT to better characterize the apparent mass lesion. We are presently conducting further analyses focused on stroke mimics in the SSTS aiming to present those findings in a subsequent publication. Regarding time from onset to thrombolysis and onset to thrombectomy, there were minor differences between the sexes, not reaching statistical significance. While detailed investigation of neuroimaging utilization and reperfusion treatments across sexes was outside the scope of this project, modern studies of sex differences in acute vessel imaging and thrombectomy are warranted, adjusting for prestroke functional level and comorbidity.

Limitations include risk of confounding by age and uncollected variables. The NIHSS was not performed in 386 patients, largely due to lack of perceived clinical indication in patients deemed on hospital arrival to have obvious mimics. It is possible that some of the 457 patients not undergoing CTA due to hospital guidelines, did have LAO stroke, and might have been eligible for EVT in healthcare systems employing other treatment criteria.

\section{CONCLUSIONS}

The SSTS had equal predictive performance for LAO stroke and EVT among men and women, despite minor sex differences in clinical characteristics in the patients undergoing ambulance transport for the suspected stroke.

\section{REFERENCES}

1. Goyal M, Menon BK, van Zwam WH, Dippel DW, Mitchell PJ, Demchuk AM, et al. Endovascular thrombectomy after large-vessel ischaemic stroke: a meta-analysis of individual patient data from five randomised trials. Lancet. (2016) 387:1723-31. doi: 10.1016/S0140-6736(16)0 0163-X

2. Mazya MV, Berglund A, Ahmed N, von Euler M, Holmin S, Laska $\mathrm{AC}$, et al. Implementation of a prehospital stroke triage system using symptom severity and teleconsultation in the Stockholm stroke triage study. JAMA Neurol. (2020) 77:691-9. doi: 10.1001/jamaneurol.20 20.0319

3. Almqvist T, Berglund A, Sjöstrand C, Eriksson E, Mazya MV. Prehospital triage accuracy in patients with stroke symptoms assessed within 6 to $24 \mathrm{~h}$ or with an unknown time of onset. Stroke. (2021) 52:1441-5. doi: 10.1161/STROKEAHA.120.033676

4. Bushnell CD, Reeves MJ, Zhao X, Pan W, Prvu-Bettger J, Zimmer L, et al. Sex differences in quality of life after ischemic stroke. Neurology. (2014) 82:922-31. doi: 10.1212/WNL.0000000000000208

5. Willers C, Lekander I, Ekstrand E, Lilja M, Pessah-Rasmussen H, Sunnerhagen KS, et al. Sex as predictor for achieved health outcomes and received care in ischemic stroke and intracerebral hemorrhage: a register-based study. Biol Sex Differ. (2018) 9:11. doi: 10.1186/s13293-018-0170-1

6. Phan HT, Blizzard CL, Reeves MJ, Thrift AG, Cadilhac D, Sturm J, et al. Sex differences in long-term mortality after stroke in the INSTRUCT (INternational STRoke oUtComes sTudy): a meta-analysis of individual

\section{DATA AVAILABILITY STATEMENT}

The raw data supporting the conclusions of this article will be made available by the authors, without undue reservation.

\section{ETHICS STATEMENT}

The studies involving human participants were reviewed and approved by Stockholm Ethics Board. Written informed consent for participation was not required for this study in accordance with the national legislation and the institutional requirements.

\section{AUTHOR CONTRIBUTIONS}

$\mathrm{AB}$ and $\mathrm{MM}$ contributed to the preparation of the dataset. MM, $\mathrm{CS}$, and EE designed the study. MM supervised the project. EW performed the statistical analysis and wrote the first draft of the manuscript. All authors contributed to manuscript revision, read, and approved the submitted version.

\section{FUNDING}

MM and CS received funding from the Innovation Fund of the Region Stockholm.

\section{ACKNOWLEDGMENTS}

The authors would thank Nils Wahlgren, MD, PhD, Karolinska Institutet, for his vision and persistence during the early years of planning of the Stockholm Stroke Triage Project. EW would thank degree project coordinator Vladimer Darsalia, $\mathrm{MD}, \mathrm{PhD}$, for his helpful input.

participant data. Circ Cardiovasc Qual Outcomes. (2017) 10:e003436. doi: 10.1161/CIRCOUTCOMES.116.003436

7. Bushnell C, Howard VJ, Lisabeth L, Caso V, Gall S, Kleindorfer D, et al. Sex differences in the evaluation and treatment of acute ischaemic stroke. Lancet Neurol. (2018) 17:641-50. doi: 10.1016/S1474-4422(18)3 0201-1

8. Labiche LA, Chan W, Saldin KR, Morgenstern LB. Sex and acute stroke presentation. Ann Emerg Med. (2002) 40:453-60. doi: $10.1067 / \mathrm{mem} .2002 .128682$

9. Gropen TI, Gokaldas R, Poleshuck R, Spencer J, Janjua N, Szarek $\mathrm{M}$, et al. Factors related to the sensitivity of emergency medical service impression of stroke. Prehosp Emerg Care. (2014) 18:387-92. doi: 10.3109/10903127.2013.864359

10. Duvekot MHC, Venema E, Rozeman AD, Moudrous W, Vermeij FH, Biekart $\mathrm{M}$, et al. Comparison of eight prehospital stroke scales to detect intracranial large-vessel occlusion in suspected stroke (PRESTO): a prospective observational study. Lancet Neurol. (2021) 20:213-21. doi: 10.1016/S1474-4422(20)30439-7

11. Madsen TE, Choo EK, Seigel TA, Palms D, Silver B. Lack of gender disparities in emergency department triage of acute stroke patients. West J Emerg Med. (2015) 16:203-9. doi: 10.5811/westjem.2014.11. 23063

12. Reddy S, Wu TC, Zhang J, Rahbar MH, Ankrom C, Zha A, et al. Lack of racial, ethnic, and sex disparities in ischemic stroke care metrics within a tele-stroke network. J Stroke Cerebrovasc Dis. (2021) 30:105418. doi: 10.1016/j.jstrokecerebrovasdis.2020.105418 
13. Govindarajan P, Friedman BT, Delgadillo JQ, Ghilarducci D, Cook LJ, Grimes B, et al. Race and sex disparities in prehospital recognition of acute stroke. Acad Emerg Med. (2015) 22:264-72. doi: 10.1111/acem. 12595

14. Cooray C, Mazya MV, Bottai M, Scheitz JF, Abdul-Rahim AH, Moreira TP, et al. Are you suffering from a large arterial occlusion? Please raise your arm! Stroke Vasc Neurol. (2018) 3:215-21. doi: 10.1136/svn-2018-000165

15. Keselman B, Cooray C, Vanhooren G, Bassi P, Consoli D, Nichelli P, et al. Intravenous thrombolysis in stroke mimics: results from the SITS International Stroke Thrombolysis Register. Eur J Neurol. (2019) 26:1091-7. doi: 10.1111/ene.13944

16. Renoux C, Coulombe J, Li L, Ganesh A, Silver L, Rothwell PM, et al. Confounding by pre-morbid functional status in studies of apparent sex differences in severity and outcome of stroke. Stroke. (2017) 48:2731-8. doi: 10.1161/STROKEAHA.117.018187

17. Cordonnier C, Sprigg N, Sandset EC, Pavlovic A, Sunnerhagen KS, Caso V, et al. Stroke in women - from evidence to inequalities. Nat Rev Neurol. (2017) 13:521-32. doi: 10.1038/nrneurol.2017.95
Conflict of Interest: The authors declare that the research was conducted in the absence of any commercial or financial relationships that could be construed as a potential conflict of interest.

Publisher's Note: All claims expressed in this article are solely those of the authors and do not necessarily represent those of their affiliated organizations, or those of the publisher, the editors and the reviewers. Any product that may be evaluated in this article, or claim that may be made by its manufacturer, is not guaranteed or endorsed by the publisher.

Copyright (c) 2021 Wiebert, Berglund, Sjöstrand, Eriksson and Mazya. This is an open-access article distributed under the terms of the Creative Commons Attribution License (CC BY). The use, distribution or reproduction in other forums is permitted, provided the original author(s) and the copyright owner(s) are credited and that the original publication in this journal is cited, in accordance with accepted academic practice. No use, distribution or reproduction is permitted which does not comply with these terms. 